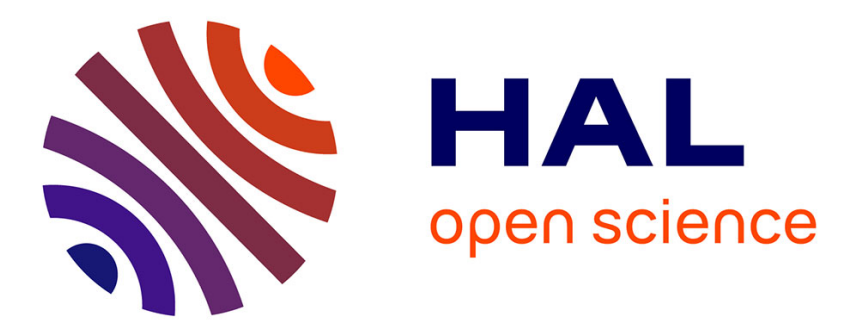

\title{
Des revendications linguistiques aux projets d'autodétermination: le cas de la Kabylie (Algérie)
}

Mohand Tilmatine

\section{To cite this version:}

Mohand Tilmatine. Des revendications linguistiques aux projets d'autodétermination: le cas de la Kabylie (Algérie). Mohand Tilmatine, Thierry Desrues. Les revendications amazighes dans la tourmente des " printemps arabes ". Trajectoires historiques et évolutions récentes des mouvements identitaires en Afrique du Nord, Centre Jacques Berque, pp.125-159, 2017, 9791092046342. hal-01824580

\section{HAL Id: hal-01824580 \\ https://hal.science/hal-01824580}

Submitted on 27 Jun 2018

HAL is a multi-disciplinary open access archive for the deposit and dissemination of scientific research documents, whether they are published or not. The documents may come from teaching and research institutions in France or abroad, or from public or private research centers.
L'archive ouverte pluridisciplinaire HAL, est destinée au dépôt et à la diffusion de documents scientifiques de niveau recherche, publiés ou non, émanant des établissements d'enseignement et de recherche français ou étrangers, des laboratoires publics ou privés. 


\section{eboos}

Centre
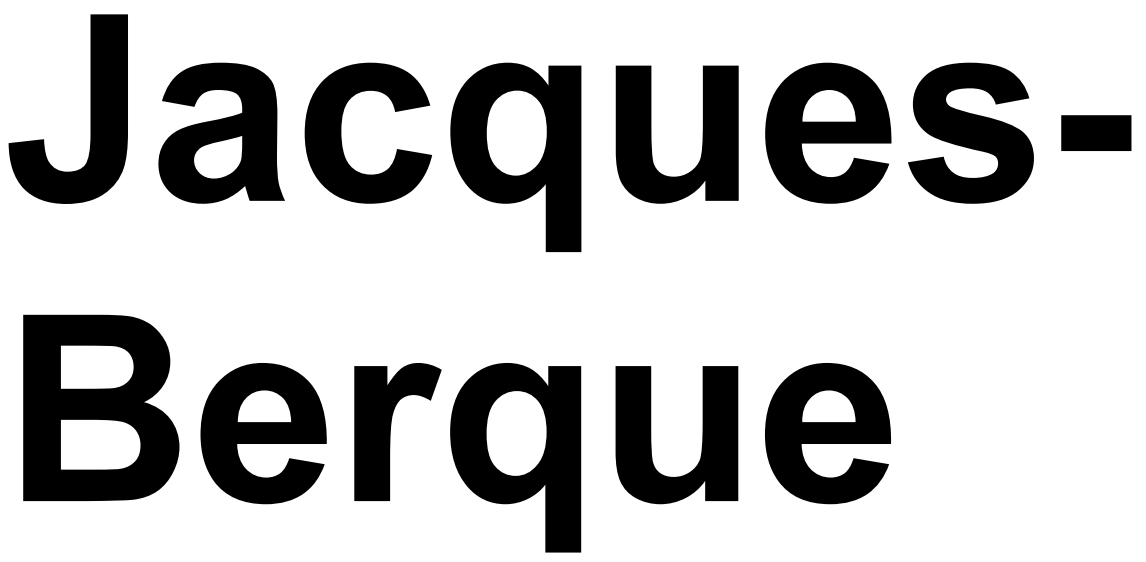

Les revendications amazighes dans la tourmente des « printemps arabes »| Mohand Tilmatine, Thierry Desrues

\section{Des}

revendications

linguistiques aux

projets

d'autodétermination : 


\section{le cas de la \\ Kabylie (Algérie)}

Mohand Tilmatine

https://orcid.org/oooo-0003-4880-4043

p. $125-159$

\section{Texto completo}

Les revendications identitaires berbères qui ont marqué ces dernières décennies la scène politique de pays nordafricains comme l'Algérie et le Maroc viennent de s'étendre plus récemment à d'autres pays de la région, où - à l'ombre des printemps dits « arabes » - le monde découvre que la Libye et la Tunisie ont également leurs Berbères qui aspirent de la même manière à la reconnaissance de leur langue et de leur culture.

2 Néanmoins, eu égard à l'étendue géographique, aux spécificités sociologiques, historiques et culturelles de chaque pays, il est clair que les contenus, l'intensité ou la forme que prennent ces revendications diffèrent d'un pays à l'autre, voire même d'une région berbérophone à l'autre.

3 Dans cette contribution, qui se limitera à l'Algérie et plus particulièrement à la Kabylie, nous nous intéresserons fondamentalement à l'analyse de l'évolution des revendications berbères - ou kabyles dans ce cas - qui passeront d'une simple demande de reconnaissance de la langue et de la culture berbères à une demande d'autodétermination. Le travail se centrera sur une tentative de reconstruction des principaux moments fondateurs qui vertèbrent les différentes phases par lesquelles passera le mouvement berbère et qui feront basculer la revendication d'un cadre déterminé pour son emboîtement dans un autre avec de nouvelles perspectives politiques mais aussi de nouvelles stratégies 
revendicatives.

4 Pour ce faire, nous nous inspirerons du point de vue méthodologique de la théorie de l'analyse des cadres, connue et largement utilisée dans l'étude des mouvements sociaux. Celle-ci préconise que les formes de mobilisation sont déterminées par l'identification subjective d'un ensemble d'individus à une " situation jugée injuste et immorale $^{2}$ ». La théorie du cadrage postule que les mouvements sociaux sont producteurs et diffuseurs de signification. Ils s'engagent dans un travail où le sens est attribué, construit, et les interprétations développées afin d'aboutir à l'adhésion et à la mobilisation des auditoires visés ${ }^{3}$. Ce processus est dénommé « cadrage » (framing) car il produit les « cadres de l'action collective ».

5 La théorie des cadres pose qu'en l'absence d'un cadre efficace, aucune mobilisation n'est possible. Pour que le mouvement puisse réaliser ses objectifs - recruter des membres, collaborer avec d'autres mouvements, mobiliser les militants en vue d'une action - il doit « encadrer »sa cause, son idéologie et son action de manière à gagner l'adhésion des différents destinataires ${ }^{4}$. Or, par « cadres de l'action », on ne se réfère pas exclusivement à ceux qui sont stratégiquement construits en vue d'une mobilisation immédiate pour une action précise. Klandermans suggère que la mobilisation à l'action est précédée par une « mobilisation de consensus » (consensus mobilization), à savoir « a process through which a social movement tries to obtain support for its viewpoint ${ }^{5}$ ». Il s'agit ainsi d'un travail de longue durée, durant lequel le mouvement propage sa critique sur une réalité problématique, tout en indiquant qu'une action collective peut aboutir à un changement social ${ }^{6}$.

6 Pour aborder ces processus, nous identifierons trois phases principales, qui marquent cette évolution depuis l'indépendance du pays :

une phase « culturaliste » qui commencera à l'époque coloniale et qui atteindra son apogée avec la 
massification de la revendication berbère durant les événements dits du " Printemps berbère » de 1980 ; une phase qui sera marquée par la radicalisation des discours et l'affrontement avec le pouvoir. Les événements de 2001 marqueront cette étape tout en entraînant certaines concessions arrachées au pouvoir central ;

une phase qui conduira à partir de 2001 à la création d'un mouvement autonomiste puis indépendantiste kabyle demandant la rupture définitive avec le pouvoir central algérien.

7 Dans chacune de ces phases, les revendications seront illustrées en mettant en exergue certaines stratégies sousjacentes à chaque étape de cette évolution. Des documents de base pour chaque période serviront de sources de références pour l'analyse des différentes phases des emboîtements ainsi que des alignements ou cadres interprétatifs mobilisés par les acteurs durant le processus revendicatif et pendant lequel le groupe remet en cause les « cadres » déjà en place pour en proposer de nouvelles significations ${ }^{7}$.

\section{La phase culturaliste : le MCB, un mouvement kabyle « algérianiste »}

\section{Localisation/périodisation : le poids de l'histoire}

8 Les origines de cette phase remontent à la première moitié du $\mathrm{XX}^{\mathrm{e}}$ siècle. Salem Chaker parlera alors d'une "veine culturaliste » incarnée par la chaîne des instituteurs et des écrivains kabyles, qui ont pour souci primordial - souvent professionnel, comme chez Boulifa, Mammeri, etc. l'étude, la conservation et la promotion du patrimoine linguistique et littéraire berbère ${ }^{8}$.

9 Pour ces auteurs, le travail culturel ne débouche pas sur une vision clairement politique, ou, si elle existe, celle-ci n'est pas assumée en tant que telle, à l'exception toutefois du fameux épisode de la crise dite « berbériste » de 1949 
pendant lequel des militants kabyles s'affrontèrent aux autres militants du mouvement national algérien sur la question de la définition de la future identité d'une Algérie indépendante.

10 En mars 1949 Ali Yahia, membre du comité directeur de la Fédération de France du Parti populaire algérien (PPA)/Mouvement pour le triomphe les libertés démocratiques (MTLD), réussit à faire voter, avec une majorité écrasante de 28 voix sur 32, une motion dénonçant le mythe d'une Algérie arabo-islamique et défend la thèse d'une Algérie algérienne. Ces événements aboutiront aux affrontements sanglants et meurtriers entre les militants dits « berbéro-nationalistes » et les partisans d'une orientation arabo-islamiste. L'assassinat, la répression et les persécutions qui s'abattent sur leurs membres les plus influents sont alors justifiés par la nécessité de faire front commun face à l'ennemi français colonisateur contre lequel le Front de libération nationale (FLN) allait déclencher la lutte armée 9 .

11 Ces événements passèrent sous la chape de plomb de la censure d'État et allaient y rester pendant des décennies après l'indépendance du pays. Toute référence au berbère et a priori à la Kabylie ou à sa spécificité était devenu tabou.

12 Nous sommes dans un cadre de diagnostic. L'injustice est évidente : malgré une participation très forte à la lutte de libération nationale, la Kabylie voit sa langue et sa culture exclues de la culture nationale et menacées par une politique d'arabisation très virulente ${ }^{10}$.

13 L'intérêt pour la culture et la langue berbères connaîtra un important mouvement de massification et se renforcera considérablement suite à un événement qui fait figure de moment fondateur du mouvement revendicatif amazigh, le Printemps berbère d'avril 1980, qui donnera naissance au Mouvement culturel berbère (MCB). Rappelons très brièvement que c'est l'interdiction d'une conférence que devait prononcer le très populaire Mouloud Mammeri à propos de son livre: Poèmes kabyles anciens (1980) - 
recueil de chefs-d'œuvre rares de la culture orale kabyle qui déclenchera les protestations et le cycle de violence et de contre-violence. Il est intéressant de souligner que c'est la répression d'un événement spécifiquement culturel qui fut le détonateur des manifestations de ce fameux Printemps berbère et des violences qui se maintinrent durant des décennies jusqu'aux événements d'avril 2001.

14 L'organisation se fera autour de la reformulation et de la reconstruction d'un autre cadre qui donnera un sens nouveau à la résistance collective face à cette répression ${ }^{11}$. On retrouvera les circonstances et le récit de ces événements narrés par un participant à ces événements ${ }^{12}$.

15 Il est important de signaler que, malgré sa localisation régionale en Kabylie, puisqu'il est parti de l'université de Tizi Ouzou, le mouvement de protestation et de revendication se propagera d'abord et parallèlement au sein de la communauté kabyle dans l'immigration pour s'étendre ensuite à d'autres régions berbérophones, surtout au Maroc. Toutefois, le contexte ou Zeitgeist de l'époque et ses contraintes politiques feront que les aspirations et revendications du mouvement berbère des années 80 se maintiendront dans une perspective « nationale » et « algérianiste ». Ceci vaut aussi bien pour les textes que pour les militants du mouvement berbère qui se sont toujours inscrits dans cet espace politique. Le poids du « nationalisme » et les tabous sur l'unité de la nation imposeront automatiquement une autocensure aux animateurs du mouvement berbère pour maintenir leurs revendications dans l'« espace national », ce qui se reflète sur le plan du discours par la revendication d'une langue amazighe appartenant à tous. Du point de vue de la théorie des cadres, on parlera "d'alignement », en d'autres termes, d'un processus interprétatif et de négociation entre les acteurs du moment qui obligera à adopter des positionnements et des ajustements en fonction des nécessités de l'environnement politique du moment.

16 Ce discours, qui se retrouve bien entendu dans les deux pays nord-africains, ne sera rompu, en Algérie, que par le 


\section{$\mathrm{MAK}^{13}$.}

\section{Du national au transnational}

17 Les revendications qui marqueront cette première phase $\mathrm{du}$ développement $\mathrm{du}$ MCB sont, pour la Kabylie, clairement énoncées dans les documents principaux du mouvement : $1^{\circ}$ le rapport de synthèse du dossier culturel adopté lors du séminaire de Yakouren du $1^{\mathrm{er}}$ au 31 août 1980, donc à peine quelques mois après les événements du Printemps berbère d'avril $1980 ; 2^{\circ}$ le rapport de synthèse du deuxième séminaire du 16 au 24 juillet 1989 à TiziOuzou $^{14}$.

18 Dans l'ensemble, le MCB revendiquait la reconnaissance de la langue et de la culture berbères mais aussi l'arabe algérien comme langues nationales ainsi que leur enseignement. C'était en effet le cadre et l'esprit de l'époque. C'est ce que nous rappelle un texte intitulé " Réflexions sur le mouvement culturel populaire en Algérie » publié dans la mythique revue Tafsut dans son premier numéro et signé par les initiales R.B. et H.S. :

« Il n'est pas besoin aujourd'hui de rappeler que c'est autour des mots d'ordre de libertés démocratiques et de langues populaires (tamaziyt et arabe algérien) que le formidable mouvement populaire s'est cristallisé ${ }^{15}$. »

19 Relevons par ailleurs la prudence extrême qui caractérise encore les positions du mouvement qui ne parle encore que d'un « mouvement populaire en Algérie »!

20 Ces revendications, d'abord locales, évolueront plus tard vers un caractère plus transnational pour toucher également la diaspora berbère en Europe et en Amérique du Nord. Les militants berbères commenceront à intervenir au niveau international dans différents cadres pour donner une certaine visibilité à leurs revendications.

21 Ainsi, des représentants feront entendre la voix des Amazighs au sein du Congrès mondial amazigh, à travers leur participation aux instances internationales de l'ONU sur les peuples autochtones, au Conseil économique et 
social des Nations Unies, du 11 février au $1^{\mathrm{er}}$ mars 2013, à la $82^{\mathrm{e}}$ session du Comité pour l'élimination de la discrimination raciale à Genève (Rapport alternatif de l'association pan-berbère Tamazgha (http://www.tamazgha.fr/), etc.

22 Cet activisme international pour faire avancer des revendications nationales est souvent défini comme un cas de glocalisation $^{16}$.

\section{Concessions et radicalisation : le « Printemps noir » et la rupture}

23 Cet ancrage de la revendication berbère et son extension, aussi bien du point du vue démographique que qualitatif, mais également son internationalisation vont conduire à certaines concessions de la part du gouvernement central d'Alger qui commencera bientôt à se rendre compte de l'étendue de la contestation et du danger qu'elle représentait pour la stabilité du pouvoir.

24 Ainsi, la multiplication des manifestations en Kabylie forcera les autorités à tolérer dans un premier temps puis à accepter l'introduction du berbère dans les universités kabyles de Tizi Ouzou (1990) et de Bejaia (1991).

25 La fameuse grève du cartable de 1995 en Kabylie débouchera sur la création du Haut Commissariat à l'Amazighité (HCA). Ainsi, le 27 mai 1995, après huit mois de boycott scolaire dans toute la Kabylie et de longues négociations entre la présidence de l'État, des représentants des deux tendances principales du MCB kabyle $^{17}$ et ceux du Mouvement culturel amazigh, chaoui et mozabite, le décret présidentiel no 95-147 du 27 mai 1995 portant création du HCA sera signé par les négociateurs des deux camps ${ }^{18}$.

26 Cette nouvelle institution, qui est un organe étatique chargé de la promotion de la langue et de la culture amazighes, répond surtout à la nécessité d'exercer un contrôle direct de l'Etat sur la gestion de la question amazighe en Algérie. En effet, les articles 2, 3 et 8 de ce 
texte décrètent que ce Haut Commissariat est directement " rattaché à la présidence de la République », que " son siège est fixé à Alger » et enfin que cet organe " est placé sous l'autorité du chef de l'État ». Il a pour missions fondamentales « la réhabilitation de l'amazighité en tant que l'un des fondements de l'identité nationale » et " l'introduction de la langue amazighe dans les systèmes de l'enseignement et de la communication » (article 4).

\section{Le berbère reconnu comme « langue de tous les Algériens ॥}

27 Un autre fait qui compte parmi les concessions du gouvernement algérien à la question berbère est sans nul doute ce que d'aucuns considèrent comme une (timide) reconnaissance de la langue berbère. En effet, le 12 mars 2002, le président Bouteflika en personne annonce la constitutionnalisation du berbère comme langue nationale tout en présentant ce geste comme entrant parfaitement dans le processus de " récupération de l'identité nationale ». Le président algérien, après toute la répression subie par les militants berbères finit même par présenter la " récupération de l'identité amazighe » comme faisant suite à celle de l'arabité en Algérie ! Le tout entrant, selon Bouteflika, dans le cadre de la reconstruction de la « personnalité nationale » à la suite des tentatives de sa destruction [par le colonialisme]. Le colonialisme étant également « coupable » de la « non-reconnaissance » du berbère en Algérie :

« Les tentatives de destruction de la personnalité algérienne ont sans doute rendu lent et complexe le processus de récupération de la personnalité nationale. Il en a été ainsi de la récupération de l'identité nationale dans son intégralité. Il en a été de même de la récupération de l'arabité de l'Algérie. Il en a été ainsi de la récupération de son amazighité. C'est donc bien de l'identité du peuple algérien dans son intégralité qu'il s'agit lorsque l'on parle d'amazighité, et le caractère national des composantes de cette amazighité ne peut donner lieu à aucune contestation 
qu'il s'agisse de la langue ou de la culture amazighe ${ }^{19}$. »

28 La langue berbère devrait donc être sans nul doute " le patrimoine de tous les Algériens », et l'amazighité, l'arabité, l'islamité mises sur le même plan « ne sont pas propres à une partie seulement des Algériens ». Pour le président de la République, cette reconnaissance ne saurait en aucun cas être perçue comme une « spécificité locale ou régionale » mais s'étend à toute l'Algérie :

« Tamazight s'entend de toutes les richesses linguistiques couvrant le territoire national aux côtés de la langue arabe, des Aurès à Kenadsa, de Bejaia à Tamanrasset, du Mzab à Tamentit, de Tebessa à Maghnia, de Azazga à Takhmart, de Bousemghoun à Tindouf et de Djanet à Bordj Badji Mokhtar $^{20}$.»

29 Sa reconnaissance n'est donc possible que si la personnalité algérienne demeure unique :

« Il est primordial de poser comme postulat que la reconnaissance $\mathrm{du}$ tamazight comme langue nationale s'inscrit dans un esprit de consolidation du processus global de restauration de la personnalité nationale unique $^{21}$. »

30 Ce discours, loin de convaincre, est perçu comme les autres concessions citées auparavant comme une énième tentative de récupération de cette revendication et de canalisation du mouvement qui demande une officialisation de la langue et de la culture. Par ailleurs, ni les activités du HCA ni son statut ne semblent permettre de mener à bon port les missions qui lui sont assignées, notamment l'introduction du berbère dans le système d'enseignement, langue qui demeure confinée au statut de langue optionnelle et surtout présente quasi exclusivement en Kabylie.

31 Nous pourrions donc penser que dans un autre cadre, plus décentralisé et plus démocratique, le principe de territorialité devrait prévaloir de manière à ce que les locuteurs d'une région déterminée puissent avoir le droit de décider sur leur territoire de leur propre politique 
linguistique, droit qui pourrait et devrait s'étendre à d'autres domaines. Selon le modèle d'analyse des principes de territorialité linguistique de Schutter, il s'agirait, dans le cas kabyle, d'une situation décrite comme la deuxième des trois options proposées :

« The second conception concerns the authority to design language regimes and says that language groups that are territorially based should be self-governing as far as this concerns linguistic affairs ${ }^{22}$. »

32 Il est clair que ni le niveau de démocratisation qu'affichent les pays nord-africains, ni les modèles centralisés à l'extrême de gouvernance qui les caractérisent ne permettent de rêver dans l'immédiat à une possible application de modèles similaires. En effet, ces derniers s'appuient sur des principes de territorialité linguistique mais surtout tirent leur légitimité de systèmes de gouvernance bien plus avancés. Cet état de fait explique d'ailleurs le type d'affrontement souvent violent qui caractérise les relations entre gouvernants et gouvernés en Afrique du Nord.

\section{Le « Printemps noir »}

33 Une date charnière dans l'évolution des revendications berbères, surtout du point de vue qualitatif, est sans doute le désormais célèbre "Printemps noir » de 2001 en Kabylie. Ces événements ont sans doute provoqué un tournant dans l'évolution des revendications identitaires dans la région et ont fait basculer celle-ci vers une nette radicalisation de ses revendications et ce dans pratiquement tous les domaines.

34 Rappelons que ces événements et ces massacres commis par la gendarmerie algérienne n'ont pratiquement pas suscité de réactions de solidarité dans les autres régions d'Algérie et ce malgré une plateforme de revendications qui demandait des mesures de démocratisation et d'amélioration sociale pour l'ensemble des Algériens.

35 Deux événements sont considérés comme les étincelles qui 
ont mis le feu aux poudres et provoqué les révoltes de jeunes Kabyles lors des célébrations - comme chaque année depuis 1980 - de la commémoration du Printemps berbère de 2001. D'abord, la mort le 18 avril 2001 d'un jeune Kabyle de 20 ans dans une caserne de la gendarmerie locale de son village à Aït Douala. Dans leur version des faits, les forces de l'ordre déclarèrent que le coup de feu qui avait provoqué la mort du jeune Massinisa était dû à un « accident par imprudence ${ }^{23}$ ».

36 Ensuite, un deuxième cas d'affrontement se produisit le 22 avril 2001, à Oued Amizour, dans la wilaya de Béjaïa, lorsque des éléments de la même gendarmerie nationale interpellèrent brutalement trois lycéens, alors qu'ils étaient avec leur classe et sous la responsabilité de leur professeur de sport, pour avoir crié des slogans interprétés par les forces de l'ordre comme « outrage à l'encontre des gendarmes de la brigade ${ }^{24} »$.

37 La spirale de violence, manifestation-répression, qui s'ensuivit provoqua plus d'une centaine de morts et des milliers de blessés parmi les jeunes manifestants kabyles, sans que les responsables n'aient jamais eu à rendre compte de leurs actes devant la justice ni qu'aucune manifestation de soutien et de solidarité provenant d'autres régions d'Algérie n'ait pu être observée.

38 Dans n’importe quel autre pays du monde démocratique, la mort de plus d'une centaine de jeunes et les blessures de plusieurs milliers de personnes par coups de feu tirés par les forces de l'ordre, et donc censées les défendre, auraient été traitées comme une tragédie nationale. En Algérie, cependant, le gouvernement n'aura même pas décrété un seul jour de deuil national.

39 Jamais auparavant les médias, pourtant habituellement prompts à défendre farouchement « l'unité nationale » du pays, n'avaient parlé aussi clairement et publiquement d'une rupture entre les Kabyles et l'État ${ }^{25}$.

40 Les jeunes Kabyles, en raison notamment du manque d'infrastructures et d'investissements dans leur région, souffrent depuis toujours du chômage. Exaspérés par le 
manque de perspectives de travail et l'absence d'avenir, ils reprochent à l'État son « mépris » (hogra) à leur égard, qu'ils comparent aujourd'hui sans ambages à celui du colonialisme français qu'ils espéraient révolu ${ }^{26}$.

41 Désormais, un grand nombre de Kabyles ne croient plus en une possible solution venant du gouvernement central ni comprennent pourquoi ils devraient continuer à s'engager et lutter pour l'ensemble de l'Algérie lorsque le reste du pays ne répond pas lorsque la répression s'abat sur eux.

\section{Répression et pression}

42 La réponse de l'État sera, comme toujours depuis l'indépendance, essentiellement répressive. Des événements très importants comme la mort du chanteur Lounes Matoub (juin 1998), l'interdiction des manifestations à Alger puis d'une manière arbitraire sur tout le territoire algérien, l'absence de mesures pour lutter contre la répression, les rapts et les violences, les morts « accidentelles » de Kabyles sous les balles des militaires algériens traitées de «bavures » et l'inflexibilité du régime et des militaires algériens ont conduit à des stratégies d'intensification avec d'incessantes manifestations de toute la société civile kabyle (médecins, avocats, étudiants, femmes, journalistes...) qui culmineront avec l'organisation de la fameuse marche sur Alger (14 juin 2001) et les graves incidents qui s'ensuivirent comme l'attaque et l'incendie d'un grand nombre de casernes de la gendarmerie en Kabylie.

43 La commission d'investigation présidée par un grand juriste kabyle, Mohand Issad ${ }^{27}$, créée sous la pression des manifestations et commandée par le président algérien pour faire la lumière sur ces événements fut très claire dans son verdict, mettant en cause la responsabilité des services de sécurité et de la gendarmerie dans les nombreuses exactions. Le rapport souligne dans ses conclusions :

« - La réaction violente des populations a été provoquée 
par l'action non moins violente des gendarmes, laquelle, pendant plus de deux mois, a nourri et entretenu l'événement: tirs à balles réelles, saccages, pillages, provocations de toutes sortes, propos obscènes et passages à tabac.

- Les gendarmes sont intervenus sans réquisition des autorités civiles comme la loi le stipule.

- La violence enregistrée contre les civils est celle d'une guerre, avec usage de munitions de guerre. »

44 Cette période du Printemps noir sera marquée par la publication d'une plateforme de revendications du mouvement citoyen kabyle et par un usage intensif des réseaux sociaux pour pallier le manque d'information sur ces événements.

45 La mémoire collective kabyle sera fortement marquée par la violence de l'État algérien. Des slogans comme Ulac Smah ulac (pas de pardon !) ou « pouvoir assassin »entretemps très répandus - refléteront ce refus de l'oubli des morts et de la répression. Le Mouvement des Aarchs de Kabylie s'inscrit néanmoins dans une perspective nationale et refuse de limiter sur le plan du discours leurs revendications au territoire de la Kabylie.

\section{La naissance d'une pensée autonomiste}

\section{"L'autonomie personnelle, locale et régionale » du FFS et du RCD}

46 Parmi les premiers projets de régionalisation ou d'autonomie régionale, il y eut d'abord celui du Front des forces socialistes (FFS), dans la clandestinité de 1965 à 1989, paru dans sa plateforme de mars 1979 intitulée : " L'alternative démocratique révolutionnaire à la catastrophe nationale. » Dans cet avant-projet, le FFS parlait de « l'autonomie personnelle, de l'autonomie locale et de l'autonomie régionale ${ }^{28} »$. Cependant, le FFS, après sa réhabilitation (1989), n'a jamais fait de ce projet un axe central de son discours ou de son action politique qui a 
toujours insisté sur sa dimension " nationale ». De fait, le rejet de l'État central est à présent si grand en Kabylie que ces deux partis légaux et nationalo-institutionnalistes, qui ont de ce fait toujours repoussé l'étiquette de «partis kabyles », prennent désormais le train de cette revendication dans le sens d'une décentralisation du pouvoir, mais sans grande conviction, l'accusation de partis « régionalistes » kabyles pesant toujours sur leur tête comme une épée de Damoclès.

47 Avec cette réaction tardive, ces partis politiques tentent de redresser la barre car ils subissent une grande baisse de popularité surtout depuis le Printemps noir, comme le démontrent l'attaque et l'incendie de quelques représentations des deux partis dans certaines régions de Kabylie.

48 Bien que le texte de présentation du nouveau projet politique du Rassemblement pour la culture et la démocratie (RCD) ne parle pas expressément d'un système fédéral ou d'autonomie régionale, le président du parti suggère dans diverses déclarations à la presse un système décentralisé, tout en considérant comme condition préalable l'inclusion des autres régions du pays dans ce processus. Dans son projet, le RCD avait proposé de créer 12 régions sur la base des spécificités de chaque partie du pays. Alger en serait la capitale. Il y aurait ainsi comme régions la Kabylie, le Titteri (Blida et périphérie), le Cheliff (Chlef et Aïn Defla), l'Oranie, la Saoura (Béchar), le Touat (Gourara), le Grand Erg oriental et l'Ahaggar'29.

\section{Les appels à l'autonomie linguistique et culturelle depuis la diaspora}

49 Malgré le tabou qui pèse sur l'« unité de la nation », l'absence d'une perspective de sortie de crise ouvrait la voie à d'autres formes de gouvernance qui remettaient en cause le système politique en vigueur. Ainsi, les premières pétitions en faveur d'une autonomie - d'abord linguistique et culturelle - de la Kabylie ne tardèrent pas à circuler. 
50 Il faudrait relever à cet égard le rôle prépondérant que joueront certaines figures du $\mathrm{MCB}$ et qui contribueront vers la fin des années 90 à la diffusion de cette idée ${ }^{30}$. Outre les publications, il y avait également des appels publics clairement en faveur de cette évolution et signés par des centaines d'intellecturels mais aussi d'autres personnes de couches sociales kabyles. Ainsi, il y eut par exemple lors du Printemps berbère de 1998 un premier appel public intitulé : "Tamazight, langue nationale de l'Algérie et langue propre de la Kabylie » et qui affirmait que la langue berbère devrait être reconnue comme « langue propre » des zones berbérophones qui le désireraient dans un cadre général d'autonomie linguistique et culturelle.

51 Un autre appel fut lancé le 29 avril à l'occasion de la rebellion de 2001 et intitulé : «Une kabylie autonome pour une Algérie démocratique », dans lequel on exigeait un statut « d'ample autonomie » pour la Kabylie, la reconnaissance de la langue berbère comme « langue propre » de la Kabylie, qui devrait disposer d'une autonomie totale dans les domaines linguistique, culturel et éducatif et finalement que l'amazigh soit une des langues nationales et officielles de l'Algérie. Ces positions partagées par divers intellectuels kabyles ont encouragé le lancement d'un débat sur le concept d'autonomie appliqué à la Kabylie. Des expériences similaires dans les pays européens, comme en Catalogne ${ }^{31}$ surtout, bien que différentes, ont certainement contribué au développement de ce débat.

\section{Le rôle de la diaspora et la référence catalane}

52 Du point de vue linguistique, des stratégies de communication accompagneront cette deuxième phase de l'évolution du discours identitaire par le choix de certains concepts, dans la mesure où l'on passera de la référence aux langues et des références communes (Berbère/Amazigh et berbérité/amazighité) à une 
référence de plus en plus claire aux spécificités kabyles.

« Nommer c'est faire exister, c'est construire. [...] Donner un nom résulte d'un processus constructiviste : c'est faire exister une réalité qui ne l'était pas auparavant, c'est homogénéiser, clôturer un ensemble de réseaux ou d'éléments à l'origine en relation les uns aux autres de manière hétérogène ${ }^{32}$. »

53 Ainsi, on passera peu à peu des « Berbères » aux «Amazighs »; du berbère ou de l'amazigh comme langue commune à l'ensemble des Berbères ou des Amazighs (pan-amazighité) à un concept emprunté à la politique autonomiste catalane : le kabyle comme « langue propre » de la Kabylie.

54 Les références ou les comparaisons avec l'expérience catalane à laquelle est emprunté le concept de « langue propre » apparaissent dès la fin des années $90^{33}$. On insiste alors plus clairement sur le fait que le concept « berbère, langue nationale », défendu par le Mouvement culturel berbère, n'est plus d'actualité et qu'il serait même dangereux « pour l'autonomie linguistique de la Kabylie » :

"Même le mot d'ordre du Mouvement culturel berbère, "berbère, langue nationale", repris par les partis politiques kabyles, paraît dangereux car aisément récupérable par l'Etat central, si son contenu n'est pas très précisément défini. Disons-le tout net, contrairement aux thèses officielles algériennes, qui explicitent une stratégie évidente de dépossession et de neutralisation, le berbère est d'abord la langue des berbérophones, car une langue sauf s'il s'agit d'une langue morte - n'existe pas en dehors de ses vecteurs humains ${ }^{34}$. »

55 Cette référence à la Catalogne n'est pas un hasard eu égard aux contacts et aux échanges qui existent entre des intellectuels ou hommes politiques kabyles et catalans (notamment Ait Ahmed, Ferhat Mehenni et Said Saadi) et qui s'est même traduite - outre la célébration de la rencontre déjà citée « Kabylie-Catalogne » en 2001 -, par la création d'un Observatoire catalan de la langue amazighe, par l'adoption d'une résolution par le parlement 
catalan en faveur de la Kabylie et condamnant la répression par l'État algérien des manifestants kabyles lors des événements du Printemps noir de 2001. L'invitation et l'accueil à Barcelone par le Pen Club catalan du seul écrivain de langue kabyle en exil, Salem Zenia, est un autre signe révélateur de ces contacts ${ }^{35}$.

56 Le concept « langue propre » sera divulgué dans plusieurs textes, déclarations, manifestes signés par des intellectuels kabyles, réunis souvent autour de Salem Chaker, linguiste et professeur à l'Institut national des langues et civilisations orientales (INALCO) et du groupe du Cercle d'étude et de réflexion sur l'autonomie de la Kabylie $(\mathrm{CERAK})^{36}$. Une déclaration dans le même sens - revenant sur les événements de 2001 - affirmait dans une déclaration du 29 avril de la même année :

« 2. La langue berbère doit être reconnue comme langue propre de la Kabylie, et la région doit bénéficier d'une autonomie totale en matière linguistique, culturelle et éducative. \ (Source : MCB-France: http://mcbrn.chez.com/declarik.html.)

\section{Le MAK et la matérialisation de la rupture}

\section{Mouvement pour l'autonomie de la Kabylie (MAK)}

57 Les commémorations du Printemps berbère de ces dernières années ont mis en évidence une évolution claire dans le paysage revendicatif kabyle: l'apparition du Mouvement pour l'autonomie de la Kabylie (MAK), qui s'impose désormais comme une force majeure supplantant des partis politiques dits kabyles et traditionnellement ancrés dans cette région comme le FFS ou le RCD ${ }^{37}$.

58 Pour la première fois dans l'histoire de l'Algérie, l'exprésident du Mouvement culturel berbère Rassemblement national (MCB-RN), Mehenni, organisait le 5 juin 2001 à Tizi Ouzou, capitale kabyle en état de siège, une conférence annonçant la naissance du MAK et exigeant une « ample » autonomie et la reconnaissance de la langue comme « langue propre de la Kabylie ». Cette 
conférence publique brisait pour la première fois ce qui était jusqu'alors un tabou : l'État-nation hypercentralisé. La classe politique algérienne, façonnée par le culte de l'Etat unique comme forme exclusive de gouvernance, est sous le choc : est-ce le début de la dislocation de l'Etat national ? La désintégration du pays est-elle en marche ${ }^{38}$ ?

59 Pour l'initiateur de ce mouvement, la Kabylie, qui se maintiendrait dans le cadre de l'État algérien, devrait disposer d'un parlement et d'un gouvernement propres et participerait au sein du gouvernement central et dans le parlement national.

60 Pour le MAK, seuls les domaines de la monnaie, de la défense nationale et de la diplomatie devraient se maintenir comme compétences de l'État central. La Kabylie devrait gérer le reste non pas à travers des représentants ou des fonctionnaires de l'État comme le wali (préfet) ou le chef de daira (circonscription) mais par le biais de représentants directement élus par les Kabyles. Une perspective qui réaffirme la nécessité de faire prévaloir la société face à l'État et non le contraire.

61 Le MAK souligne sa démarcation des projets de « régionalisation positive » et de « refondation nationale » en argumentant par le fait qu'il exprime plus concrètement et plus ouvertement ses idées. Par ailleurs, le RCD et le FFS revendiquent la régionalisation à l'échelle nationale alors que le MAK se limite au territoire kabyle. Il n'a aucune ambition nationale ni prétend imposer aux autres régions ce qu'il réclame pour le territoire kabyle. " Le fédéralisme », estime Ferhat Mehenni, « est une autre étape de notre avenir, il suppose que l'ensemble des régions du pays le revendique au même titre que la Kabylie. Nous ne pouvons plus attendre jusqu'à ce que toutes les régions soient d'accord pour accéder à la maîtrise de notre destin ${ }^{39} »$. Chaque région, ajoute Ferhat Mehenni, « pourrait développer son idée d'autonomie, mais il ne nous revient pas à nous de nous substituer à eux en cela ${ }^{40} »$.

62 Cette démarche s'inscrit parfaitement dans ce que la 
théorie des cadres définit comme une « construction sociale d'une représentation commune de la situation ». La mobilisation se produit dès lors d'abord autour d'un diagnostic de la situation (diagnosis framing) : échec des expériences passées du RCD et du FFS, mais aussi du modèle central de l'État-nation ; suivi d'une phase de pronostic (pronostic framing), donc l'identification et l'attribution des responsabilités, dans ce cas : l'État, sa politique mais aussi les partis «algérianistes kabyles »; enfin, la phase de motivation pour l'action (motivational framing), développant un discours de prise en charge de manière autonome et indépendante du « destin de la Kabylie $^{41} »$.

63 Le MAK insiste, chaque fois que l'occasion lui est donnée, sur le fait qu'il s'agit d'un mouvement pacifique et que ses actions seront purement politiques et se prononce contre tout type de solution aventurière. Il pense influer sur le gouvernement en organisant un référendum sur le sujet.

64 Les dernières manifestations organisées par le MAK ces dernières années sont souvent une véritable démonstration de force et de capacité de mobilisation de la part d'un mouvement qui, depuis sa première apparition en juin 2001, demeure sans existence officielle ni, bien entendu, sans les structures organiques nécessaires. Il est également clair qu'un tel mouvement ne saurait prétendre à une reconnaissance dans le cadre de la Constitution en vigueur. Bien au contraire, le gouvernement aurait suffisamment d'arguments juridiques pour interdire et empêcher ses activités, situation qu'il exploite d'ailleurs souvent par la répression (Le Matin du 13 janvier 2010) des militants du MAK et qui peut prendre différentes formes comme l'intimidation, divers types de "chicanes" comme la fermeture des négoces appartenant aux militants du MAK, l'emprisonnement ou la détention provisoire, comme ce fut le cas lors du dernier congrès du MAK lorsque plus de 300 congressistes furent retenus pour les empêcher d'arriver à temps sur les lieux du congrès.

65 Malgré ces difficultés et indépendamment de son véritable 
impact sur le terrain, il ne fait aucun doute que le simple fait d'exister virtuellement - par exemple à travers son site Web - semble avoir provoqué un grand intérêt et une grande illusion dans la population et favorisé une très large diffusion de ses idées dans toute la Kabylie mais aussi dans la diaspora en Europe et en Amérique du $\mathrm{Nord}^{42}$. Un processus qui impose désormais sur l'échiquier politique du pays et de manière durable le débat sur l'autonomie de la Kabylie. Le processus se consolide avec la publication de documents comme le «Projet pour l'autonomie de la Kabylie $^{43}$ » ou le « Projet pour un État kabyle » adopté à la conférence nationale des cadres du MAK le 24 janvier 2014 à Smaoun en Kabylie ${ }^{44}$.

66 L'expansion du MAK est telle que même des journaux nationaux peu suspects de sympathie à son égard le perçoivent très souvent comme un mouvement séparatiste et comme le plus mobilisateur de la Kabylie ${ }^{45}$. D'autres, comme El-Khabar, le plus fort tirage d'Algérie, n'hésitaient pas à se demander, dans leur édition du 14 avril 2016, si l'afflux des ministres algériens en cette période qui précède la $36^{\mathrm{e}}$ commémoration du Printemps berbère de Kabylie (2016) était dû au fait que le gouvernement avait peur du MAK ou si c'était en raison de l'expansion de ce mouvement politique en Kabylie $^{46}$.

\section{De l'autonomie à l'autodétermination}

67 Le MAK est-il le résultat d'une continuité directe et logique des revendications berbères ? Cette version est reprise par le discours autonomiste qui présente les différentes phases par lesquelles est passée la revendication berbère comme des étapes logiques et progressives d'une même revendication. La proclamation du MAK serait selon les autonomistes kabyles l'aboutissement d'un long processus qui commença par la fameuse crise dite «berbériste » de 1949, en passant par l'insurrection du FFS de 1963, le Printemps berbère d'avril 1980 de Tizi-Ouzou, la création du RCD et le retour du FFS en 1989, la grève du cartable de 
1995, jusqu'aux morts tombés lors du Printemps noir de 2001, prélude à la création du MAK en juin $2001^{47}$.

68 Il serait probablement plus juste de considérer qu'il s'agit là d'un processus historique qui est passé par une série d'événements et de revendications identitaires berbères, certes, mais qui ne participaient pas tous de l'objectif actuel du MAK. Ces événements se sont tous déroulés dans des contextes spécifiques, mais pas forcément indépendants les uns des autres. Mais il est certain que ni les « Berbéro-matérialistes » des années 40, ni le Mouvement culturel berbère de 1980 n'avaient pour objectif une autonomie ni encore moins une hypothétique souveraineté de la Kabylie.

69 Néanmoins, il est certain aujourd'hui que la création d'un mouvement pour l'autodétermination de la Kabylie est perçue par une grande partie de ses partisans comme une évolution logique.

70 Le rejet global du pouvoir et des forces politiques traditionnelles algériennes n'en est qu'un aspect. La réorientation stratégique est davantage le fruit de l'expérience empirique. En effet, les autonomistes et souverainistes kabyles, après s'être investis dans la démocratisation du pays pour la reconnaissance de l'amazigh et de l'arabe algérien, se rendent compte que tous ces efforts n'ont pas abouti aux résultats attendus et commencent à se détourner de certaines idées, jusque-là fortement ancrées dans les consciences des militants, comme celle de croire en un amazigh commun, pour focaliser depuis 2001 leurs revendications sur la seule Kabylie.

71 Le MAK a annoncé son intention de passer la vitesse supérieure en passant de l'objectif d' « autonomie » à celui d' « autodétermination ». La réunion du conseil national du MAK en session ordinaire le 4 octobre 2013 dans le village d'Ath Hamdoune, en Kabylie, aboutit à l'annonce que le MAK passera de l'autonomie à l'autodétermination et s'appellera désormais « Mouvement pour l'autodétermination de la Kabylie ». Cette évolution est 
motivée selon les congressistes par le fait que le concept de droit à l'autodétermination présenterait au moins deux avantages, que la base militante et le citoyen kabyle peuvent comprendre aisément :

72 1. Le droit à l'autodétermination est tout simplement un exercice de démocratie. C'est un référendum par lequel chaque citoyen est appelé à s'exprimer en son âme et conscience sur le mode de gouvernance qu'il souhaite pour le peuple.

73 2. Il recouvre toutes les possibilités qui peuvent se présenter pour l'avenir politique de la Kabylie, aussi bien le statu quo que l'évolution vers une gouvernance kabyle dans le cadre interne de l'Algérie (autonomie) ou dans un cadre externe, autrement dit l'indépendance.

74 À travers ce droit à l'autodétermination, le MAK dit chercher à rendre la parole au peuple kabyle au moment opportun afin qu'il soit en mesure de choisir librement le statut politique qui lui sied (Le Matin du 6 octobre 2013). Cette décision a été rendue publique dans un communiqué en date du 28 octobre 2013 et repris, entre autres, par l'agence de presse du MAK, Siwel ${ }^{48}$.

75 Il est intéressant de relever que le droit international, notamment à travers l'ONU, reconnaît ce droit qui avait été abondamment utilisé par les pays encore sous dépendance coloniale dans les années 50 et 60 du siècle dernier. Ce droit continue par ailleurs de constituer un recours pour un certain nombre de nations sans État, dont certains très médiatisées comme les Québécois, les Écossais et surtout dernièrement les Catalans. Toutes ces nations aspirent à obtenir l'indépendance de leurs pays respectifs à travers l'organisation d'un référendum.

76 Il est clair que la situation diffère énormément selon qu'il s'agit de pays à longue tradition démocratique comme le Canada ou la Grande-Bretagne. Même l'Espagne, qui pour le moment ne peut pas encore être qualifiée de démocratie de haute facture, présente une situation qui est aux antipodes de celle de l'Algérie, considérée comme foncièrement anti-démocratique. Dans l'état actuel des 
choses, le MAK n'a pratiquement aucune chance d'obtenir gain de cause. Pourtant, son travail a obtenu de grands résultats ces dernières années en devenant, sans aucun doute, le mouvement politique le plus mobilisateur de la Kabylie et ce, aux dépends, par exemple, du RCD ou surtout du FFS. Ce dernier n'hésite pas d'ailleurs à accuser le gouvernement algérien, à travers les scandales de corruption qui l'ont affecté, d'être à l'origine de l'avancée du $\mathrm{MAK}^{49}$. Il est intéressant de noter que ces arguments (l'intransigeance de l'État comme fabrique des indépendantistes) rappellent ceux avancés en Espagne pour expliquer l'avancée des indépendantistes en Catalogne ces dernières années ${ }^{50}$.

77 Outre les médias algériens, les réseaux sociaux ont relevé que les manifestations du MAK dans la diaspora (Paris et Montréal) et celles du 20 avril 2016 en Kabylie (Bouira, Bejaïa et surtout Tizi-Ouzou) ont mobilisé une véritable marée humaine ${ }^{51}$. Même si les médias nationaux algériens ont essayé de minimiser ou de taire ces événements, d'autres, émettant d'Europe, comme la Magharibia ou France 24 ont même offert au leader du MAK une tribune pour qu'il présente ses idées (Siwel, 19 avril 2016).

78 Ce saut qualitatif du MAK n'est certainement pas dû au hasard mais au grand travail effectué notamment par les militants en Kabylie, par leur charismatique président, Bouaziz Ait-Chebib, mais aussi grâce à une stratégie de communication multiforme dont un travail de lobbying diplomatique mené par le gouvernement provisoire de Kabylie (GPK) sous la direction de Ferhat Mehenni.

79 Il est également intéressant de relever que le MAK commence à avoir un certain impact sur la politique régionale des États nord-africains. Depuis le Maroc, on demande aux Algériens « d'appliquer le droit à l'autodétermination »- que ces derniers défendent dans le cas des Sahraouis - également aux «Kabyles et au Sud algérien $^{52} »$. Le représentant du Royaume du Maroc a même exprimé dans le cadre de la $70^{\mathrm{e}}$ session de l'Assemblée générale de l'ONU, le 27 octobre 2015, le 
soutien de son pays au droit du peuple kabyle à l'autodétermination ${ }^{53}$, même s'il est clair que cette position s'inscrit dans le cadre des escarmouches régulières entre les deux pays à propos de la question du Sahara occidental.

80 Du point de vue strictement idéologique, le discours du MAK s'insère largement dans la globalisation, la modernité dans laquelle il aspire à une redéfinition du rôle de certains indicateurs religieux, culturels ou idéologiques qui servent pour la formation et la constitution d'une nouvelle conscience identitaire kabyle. Ainsi, le vieux concept d'ethnicité laisse le champ au concept de " langue propre », emprunté à la sociolinguistique catalane, comme élément qui vertèbre et cimente le projet kabyle. Le choix, ouvertement laïc, implique une distanciation par rapport au rôle prépondérant que joue actuellement l'islam dans l'Algérie post-indépendance comme religion d'État. La religion cesserait de fonctionner comme un trait définitoire de l'identité kabyle pour s'ouvrir à la diversité religieuse (le MAK a durement critiqué les attaques contre les églises évangéliques en Kabylie).

\section{Retour à l'autonomie. Naissance du Rassemblement pour la Kabylie (RPK)}

81 Malgré toutes ces avancées, ou peut-être en raison de son succès, notamment auprès des jeunes, la direction du MAK/ANAVAD fera, dans sa lancée, un autre pas lors d'un discours devant les militants à Montreuil, en France, le 25 septembre 2016. Son président, Ferhat Mehenni, annoncera une " autre lecture du Troisième congrès du MAK » du 26 février 2016 :

« Le Projet d'un Etat kabyle (PEK) a été réécrit de manière à écarter définitivement toute autre option pour notre Mouvement en dehors de l'indépendance du peuple kabyle. Le droit à l'autodétermination de la Kabylie qui reste notre objectif stratégique est bel et bien redéfini comme celui de notre indépendance et non celui d'un autre statut ${ }^{54}$. »

82 Un pas supplémentaire qui creusera probablement les 
différences entre le président du GPK, en exil, et celui du MAK, en Kabylie, et aboutira à la démission de ce dernier le 16 novembre $2016^{55}$. Le retrait de ce personnage charismatique et apprécié par les militants, qui avait été reconduit par le dernier congrès du MAK, entraînera un certain nombre de démissions et de grands débats entre les militants sur les réseaux sociaux et ne manquera pas de provoquer une scission au sein des troupes du MAK, partagées désormais entre partisans d'une large autonomie et partisans d'une indépendance kabyle, le tout dans un contexte de pression et de répression des militants actifs en Kabylie, qui souffrent d'intimidations de toutes sortes.

83 Loin de se ressaisir, la direction du MAK radicalise sa position en accélérant la cadence avec la création d'un parlement en exil à Montréal, au Canada ${ }^{56}$, et en présentant un projet de nomination « d'ambassadeurs » du GPK dans le monde ${ }^{57}$. C'est dans ce contexte que sera annoncée la création d'un nouveau mouvement politique sur la scène kabyle le 26 février 2017: le Rassemblement pour la Kabylie (RPK) ${ }^{58}$.

84 Ce parti est né d’une série de réunions qui ont regroupé des autonomistes kabyles - non indépendantistes - qui résument leurs positions dans un « Manifeste kabyle » publié la première fois dans la presse algérienne en janvier $2014^{59}$. La différence fondamentale entre cette nouvelle formation politique et le MAK réside dans le fait que le RPK s'inscrit dans la légalité et dans un espace national algérien. La Kabylie, selon le coordinateur de ce mouvement, « fait partie de l'Algérie et doit y rester ${ }^{60}$ ».

85 Il ne fait pas de doute que le contexte favorise ce projet du RPK. Ce dernier profitera sans doute du fait que le choix exclusif du MAK pour l'indépendance laisse " orpheline » sa frange autonomiste et qui se sentait exclue par cette réorientation de la présidence du GPK/MAK. Le RPK pourra donc « hériter » du travail formidable de mobilisation fait par le MAK depuis sa création en 2001 pour proposer aux exclus idéologiques de ce mouvement un cadre et un espace d'action que le MAK avait 
volontairement supprimé.

\section{L'État-nation comme unique forme de gouvernance: la fin d'un tabou?}

86 L'officialisation de la langue berbère en Algérie, introduite lors de la dernière modification constitutionnelle en décembre de 2015 et adoptée par le Parlement algérien le 7 février $2016^{61}$, n'a guère changé les choses pour le MAK qui considère cette revendication comme dépassée. En revanche, la naissance du MAK et surtout l'extension du mouvement sont en train de provoquer un débat sur le système de gouvernance en Algérie. Des journaux de première importance ne peuvent plus ignorer son impact et commencent à ouvrir leurs colonnes aux animateurs du Mouvement comme Ferhat Mehenni ou l'ex-président du MAK, Bouaziz Ait Chebib ${ }^{62}$.

87 Peu à peu, le débat s'ouvre - encore timidement, certes sur d'autres options politiques et formes de gouvernance comme par exemple celle d'une Algérie fédérale ${ }^{63}$, même si le thème demeure largement tabou dans la presse algérienne et les débats publics. Même si on n'ose pas encore la nommer, l'autonomie régionale est citée comme une possibilité de gouvernance par des journalistes algériens nationalistes comme Maamar Farah :

« Il est absolument inconcevable que, sur tous les modèles d'organisation étatique qui ont prouvé leur solidité et leur efficience, nous ayons choisi celui, jacobin, qui convient peut-être à la France mais pas à un pays aussi immense dans sa géographie et aussi divers dans ses ethnies et ses cultures. L'Espagne, l'Allemagne, les Etats-Unis, la Russie, la Chine sont des modèles que nous avons, hélas, refusé de suivre $^{64}$. »

88 Certaines personnalités publiques, kabyles également, comme l'avocat et vieux militant Maître Ali Yahia, président de la Ligue des droits de l'homme, déclare sans ambages qu'il faut reconnaître le mouvement pour l'autonomie de la Kabylie ${ }^{65}$. Sans toujours apporter un 
appui explicite à la démarche du MAK, certaines personnalités publiques très importantes comme le chanteur kabyle Idir demandent au moins d'écouter ses propositions sans les diaboliser ${ }^{66}$. Peu à peu, des journalistes commencent à se poser la question de savoir s'il ne faut pas également chercher les maux ailleurs que dans le « séparatisme » du fondateur du MAK. C'est ce qu'exprime le mieux la journaliste Sarah Haidar (2015) dans le journal Le Soir d'Algérie ${ }^{67}$ :

" Il existe un besoin, plus visible et moins censuré en Kabylie, d'une libération des mœurs et d'une sortie plus que jamais nécessaire de l'aliénation dogmatique gaiement entretenue par les pouvoirs centraux. Cela peut paraître insignifiant, mais il y a bien une raison pour qu'une bonne partie de la société algérienne " mécréante » prenne la route vers ce " territoire » afin d'y goûter aux libertés et aux plaisirs simples ou d'y " manger le Ramadan » sans être terrorisée par une éventuelle descente de police suivie d'un procès ! Cette raison est que la Kabylie, qu'on le veuille ou non, est la seule région à résister encore au double diktat social et religieux, à être plus ou moins épargnée par la victoire idéologique des islamistes et à porter en elle les germes d'une sécularisation possible. Laquelle est évidemment impensable dans le giron d'un Etat qui impose "sa" religion à l'intégralité de ses citoyens et qui a prouvé, au fil de l'histoire, sa totale imperméabilité au changement. Entre cette Algérie soumise et confortablement installée dans le dogmatisme et l'inquisition et cette Kabylie où le dialogue est possible, Ferhat n'a fait que choisir. On ne peut continuer éternellement à faire passer ce choix pour un crime impardonnable. Le vrai débat surviendra un jour : l'histoire n'en sera que plus logique ! »

89 Aujourd'hui, même des hommes politiques - non Kabyles - comme Noureddine Boukrouh (2016) en arrivent à qualifier l'Algérie de « fausse nation ${ }^{68}$ ». L'auteur, qui évite soigneusement de mentionner le MAK dans tout son texte, commence en rappelant que :

« Ayant été mal faite, l'Algérie est appelée à être refaite. On 
ne sait quand ni à quel prix, mais presque tout devra être refait un jour. »

\section{Conclusion}

90 Eu égard à l'évolution qualitative des revendications, il semble assez clair qu'une des premières conclusions à tirer est que l'option souverainiste a été provoquée, ou en tous cas renforcée, par l'inflexibilité du gouvernement central et par la gestion catastrophique des revendications amazighes depuis le début des protestations massives d'avril 1980.

91 L'avancée du MAK a aujourd'hui des conséquences sur la prise de conscience de la question amazighe à pratiquement tous les niveaux. Il ne fait pas de doute que l'officialisation de la langue amazighe en Algérie participe d'une tentative de désactivation de "l'effet MAK » en Kabylie. Aujourd'hui, l'État assume publiquement l'amazighité de l'Algérie, et bien peu de partis refusent de nos jours son officialisation. Même des islamistes comme Ali Benhadj déclarent que c'est l'État algérien qui crée les crises et que «si le gouvernement algérien avait pris en compte - en son temps - les revendications amazighes nous n'en serions pas là ${ }^{69}$ ».

92 Aujourd'hui, des quotidiens algériens de tirage national comme Le Soir d'Algérie revoient leur copie en reconnaissant désormais que " le MAK est en train de gagner du terrain en Kabylie et [que] ses idées deviennent progressivement discutables après avoir été longtemps haïssables ${ }^{70} »$.

93 Après plus de quinze ans d'existence, l'option exclusivement « souverainiste » du GPK/MAK semble entrer dans une phase de confusion. La répression, le manque de perspective d'une solution pacifique et consensuelle sur le terrain ainsi que les doutes sur les risques qu'induisent des positions de rupture totale pour l'intégrité et la paix en Kabylie semblent ouvrir la voie à d'autres options, qui semblent plus « réalistes ».

94 L'irruption du RPK en février 2017, qui aspire à un statut 
particulier de la Kabylie tout en se réclamant de l'espace national algérien, ouvre un nouvel espace de débat qui pourrait la « réconcilier » avec une opposition « algérienne » démocratique - certes encore infime - mais qui pourrait voir dans ce discours une chance de règlement de la «question kabyle » tout en coupant les ailes aux indépendantistes du GPK/MAK.

95 La naissance du RPK vient du point de vue stratégique occuper l'espace délaissé par le MAK et profitera du formidable mouvement de mobilisation dont les autonomistes et les indépendantistes kabyles ont fait la démontration lors des manifestations d'avril 2016. La création d'un parti autonomiste, mais légaliste et s'inscrivant dans une perspective algérienne, met en mauvaise posture le gouvernement actuel qui aura à présent plus de difficultés à justifier son refus de donner un agrément à un parti politique qui se déclare algérien mais proposant une autre forme de gouvernance : un type de fédéralisme asymétrique, encore à définir et en tous cas une refondation de l'État-nation que beaucoup ne cessent d'exiger et de considérer comme le problème fondamental de l'Algérie ${ }^{71}$. Certes, les lois algériennes ne permettent pas dans les circonstances actuelles d'espérer un agrément pour ce nouveau parti. Ses idées sont encore loin d'obtenir l'aval d'une majorité au sein du parlement ou même du peuple algérien, trop longtemps formaté au discours nationaliste, unanimiste et monolithique. Mais c'est certainement une légère avancée, d'autant plus que l'article 5 de l'ordonnance 1997 sur les partis politiques, qui interdisait la création de partis politiques sur des bases religieuses, linguistiques ou régionales ${ }^{72}$, a été réduit dans le texte révisé de $2012^{73}$ à la seule religion, même si ces contraintes réapparaissent sous une autre forme dans l'article 24. La balle est plus que jamais dans le camp gouvernemental, qui doit désormais affronter le renforcement de fait des rangs autour des revendications spécifiquement kabyles.

96 Ce mouvement gagnera-t-il également les autres pays de 
Tamazgha ? Difficile de le dire tant les conditions diffèrent d'un pays à l'autre. On constate que le MAK a commencé à faire des émules dans d'autres régions berbérophones, aussi bien en Algérie (Mzab, Chawiyas) qu'au Maroc (Rif, Grand Souss), voire même en Libye où, après les autonomistes de la région est du pays, les Berbères commencent à parler également d'autodétermination ${ }^{74}$. $\mathrm{Au}$ Maroc, le Mouvement pour l'autonomie du Rif s'appuie notamment sur l'expérience de la République du Rif, qui a fonctionné comme un «Etat » indépendant entre 1920 et $1926^{75}$, pour développer dans ses discours la revendication du rifain en lieu et place de l'amazigh standard développé par l'IRCAM ${ }^{76}$. Difficile d'évaluer la force de la tendance autonomiste rifaine, probablement très limitée jusqu'à présent. Cette perspective se voit, cependant, considérablement renforcée depuis la mort horrible d'un jeune poissonnier rifain, Mohcine Fikri (31 ans), broyé dans une benne à ordures le 20 octobre 2016 à $\mathrm{Al}$ Hoceima, en plein cœur du Rif. La colère suscitée par la mort tragique de ce jeune et modeste travailleur n'est pas sans rappeler celle de Mohamed Bouazizi, jeune Tunisien qui s'était immolé en Tunisie le 4 janvier 2011 et dont la mort annonça le « Printemps arabe ».

97 Dans les deux cas, ces morts seront suivies par de très fortes mobilisations populaires qui exprimeront leur colère face à cette injustice. Nous sommes en plein cadre diagnostique : " le problème », sa cause et son ampleur sont définis et caractérisés, et la responsabilité attribuée : l'absence de justice et un Makhzen qui maltraite le Rif.

98 La forte répression qui s'est abattue sur les participants, notamment depuis l'arrestation de leur figure visible, Nasser Zefzafi, le 29 mai 2017, accusé « d'atteinte à la sécurité intérieure de l'État ${ }^{77}$ », ne fait que renforcer ce sentiment. Les manifestations et les actions de protestation s'articulent autour d'un « Mouvement populaire du Rif », créé autour de Zefzafi et connu localement sous le nom de Hirāk Arrīf (al Hirāàk aš-šasbi fi Rìf) en arabe et Amussu agherfan $n$ Arrif en amazigh. Les objectifs sont formulés, 
des solutions proposées et des méthodes d'action et d'adaptation à la répression sont prescrites aux manifestants.

99 Bien que les revendications de ce mouvement soient jusqu'à présent strictement d'ordre économique et social, il n'en demeure pas moins qu'un cocktail explosif de sentiments de hogra est présent depuis très longtemps dans la région. Ces sentiments sont alimentés par la sensation d'abandon par l'État central depuis l'indépendance du pays, par les douloureux souvenirs des répressions de la fin des années 50 et surtout de l'époque de la République d'Abdelkrim al-Khettabi. La conjonction de ces facteurs et du particularisme linguistique et culturel renforce les sentiments d'une appartenance commune et d'une « spécificité rifaine » qui se manifeste par des drapeaux amazighs et de la République du Rif, fièrement brandis lors des manifestations dans le Rif et dans la diaspora, mais aussi et surtout par la construction d'un discours de représentation de la situation comme injuste et discriminatoire. L'accord sur le diagnostic de la situation est construit pour le moment autour de revendications économiques et sociales. Néanmoins, tous les ingrédients semblent converger vers le renforcement d'une revendication identitaire qui semble vouloir aller bien audelà de la simple reconnaissance de l'amazigh comme langue officielle au Maroc.

\section{Bibliografía}

Abelfattah Lami N., " Du mythe de l'isolat kabyle », Cahier d'études africaines, $\mathrm{n}^{0}$ 175, 2004, p. 507-531.

Abrous D., " Le Haut-Commissariat à l'Amazighité ou les méandres d'une phagocytose », Annuaire de l'Afrique du Nord, 34, Paris, CNRS éditions, 1995, p. 583-590.

ACHAB R., La Néologie lexicale berbère (1945-1995), ParisLouvain, Peeters, 1996. 
Bellal S., « Dutch disease et désindustrialisation en Algérie, une approche critique ", Revue du chercheur, 2013, p. 1-13.

BELlil R., Hachi S., " Réflexions sur le mouvement culturel populaire en Algérie : la culture et le centralisme », Tafsut, no 1, Tizi Ouzou, Cité universitaire, 1981, p. 13, [Revue clandestine du Mouvement berbère, série "normale"].

BENFORD R.D. et al., " Processus de cadrage et mouvements sociaux : présentation et bilan ", Politix, 3/99, 2012, p. 217-255.

BENFORD R.D., SNOW D.A., « Framing processes and social movements: an overview and assessment ", Annual Review of Sociology, 26, 2000, p. 611-639.

BenNis S., " Société civile et nouveaux paradigmes conceptuels : le concept de hogra », à consulter sur le site $\mathrm{du}$ Centre d'études et de recherches en sciences sociales http://www.cerssma.org /new/index.php?option=com_content\&view=article\& $\mathrm{id}=279$ : societe-civile-et-nouveaux-paradigmesconceptuels--le-concept-de-l-hogra-r\&catid=86:articlebennis\&Itemid=108, mercredi 9 janvier 2013 [consulté le 5/07/2017].

Bouteflika A., Discours à la Nation (Alger, mardi 12 mars 2002), [en ligne] URL: http://www.el-mouradia.dz /francais/president/recherche/presidentrech.htm [consulté le 12/11/ 2015].

CANut C., " À la frontière des langues : figures de la démarcation ", Cahiers d'études africaines, n ${ }^{0}$ 163-164, XLI-3-4, 2001, p. 443-463.

Castellanos C. et al., (dir.), Actes de la Rencontre Kabylie-Catalogne : identités nationales et structures étatiques dans le contexte méditerranéen, Barcelone, 13 et 
14 septembre 2002, Paris, Editions berbères, 2008.

Chaker R., "Journal des événements de Kabylie (marsmai 1980) », Les Temps modernes, Algérie : espoirs et réalités, $\mathrm{n}^{\mathrm{o}}$ 432-433 (juillet-août 1982), p. 383-438.

Chaker S., " L'affirmation identitaire berbère à partir de 1900 : constantes et mutations (Kabylie) », Revue de l'Occident musulman et de la Méditerranée, 44/1, 1987, p. 13-34.

Chouli L., " Les mouvements sociaux de 1998 et $2011 \mathrm{au}$ Burkina Faso comme indices et réponses à la crise de la représentation politique ", Le Retour de la question politique : crise de la représentation et luttes démocratiques en Afrique, $4^{\mathrm{e}}$ Colloque international de Dakar, 22-24 mai 2013 (à consulter sur : essai.gabrielperi.fr/IMG/pdf/7-lila_chouli.pdf [consulté le 3/07/2017].

Contamin J.G., « Cadrages et luttes de sens », dans Penser les mouvements sociaux : conflits sociaux et contestations dans les sociétés contemporaines, sous la dir. Olivier Fillieule et al., Paris, La Découverte, 2010, p. 56-75.

De Schutter H., « The Linguistic Territoriality Principle, A critique », Journal of Applied Philosophy, vol. 25, No. 2, 2008, p. 105-120.

Front des forces socialistes, L'Alternative démocratique révolutionnaire à la catastrophe nationale : avant-projet de plateforme politique, brochure interne, mars 1979. Disponible sur : https://leseditionsachab.wordpress.com /2016/05/20/ffs-1979-avant-projet-de-plateformepolitique-lalternative-democratique-revolutionnaire-a-lacatastrophe-nationale/ [consulté le 7/06/2016].

GeIsSER V., « Trop diplômés pour être honnêtes : la hogra des immigrés en "col blanc” », Migrations Société, vol. 138, $\mathrm{n}^{\circ}$ 6, 2011, p. 3-12. 
Guennoun A., Chronologie du mouvement berbère 1945-1990 : un combat et des hommes, Alger, Casbah Editions, 1999.

ILIKOUD O., "FFS et RCD : partis nationaux ou partis kabyles ?», Revue des mondes musulmans et de la Méditerranée, [en ligne], 2011/111-112 | mars 2006, mis en ligne le o8/12/ 2011, URL : http://remmm.revues.org/2870 [consulté le 14/o7/2015].

IsSAD M., Rapport préliminaire de la Commission nationale d'enquête sur les événements de Kabylie (juillet 2001), [2001], [en ligne] URL: http://www.algeriawatch.de/farticle/revolte/issad_rapport.htm [consulté le 16/10/2015].

La Question amazighe : interrogations actuelles ... Actes de la table ronde organisée par le MCB-France, Paris 21 avril 1996, Paris, Publications MCB-France.

Kabylie : l'autonomie en débat, Actes du séminaire d'Ecancourt-France, 1-3 mars 2002, Paris, Trèfle Communication.

Klandermans B., Oegema D., " Potentials, Networks, Motivations, and Barriers: Steps Towards Participation in Social Movements ", American Sociological Review, 52, 1987, p. 519-531.

Klandermans B., « Mobilization and Participation: Social Psychological Expansions of Resource Mobilization Theory ", American Sociological Review 49, 1984, p. 583-60o.

MAdariaga M.R. de, España y el Rif. Crónica de una historia casi olvidada, Melilla, La biblioteca de Melilla, tercera edición, 2008.

Mehenni F., Algérie : la question kabyle. Essai, Paris, Editions Michalon, 2004. 
OuERDANE A., "Un conflit à plusieurs faces: "la crise berberiste" de 1949 ", Revue de l'Occident musulman et de la Méditerranée, $\mathrm{n}^{0}$ 44, 1987, p. 35-47.

OUERDANE A., La Question berbère dans le mouvement national algérien, 1926-1980, Québec, Sillery ; Paris, Septentrion, 1990.

Robertson R., " Glocalization: time and space and homogeneity-heterogeneity ", dans Global Modernities, sous la dir. de M. Featherstone et al., London, Thousand Oaks, New Delhi, Sage Publications, 1995, p. 25-43.

SNOw D.A., " Analyse des cadres et mouvements sociaux », dans Les Formes de l'action collective, sous la dir. de Daniel Cefaï et Danny Trom, Paris, Éditions de l'EHESS, 2001, p. 1-21.

Tilmatine M., « L'Etat-nation face à la revendication berbère : quel(s) modèle(s) pour l'Algérie ? ", La Question amazighe : interrogations actuelles, Actes de la tableronde organisée par le MCB-France, 21 avril 1996, Paris, MCB-France, 1996a, p. 33-44.

Tilmatine M., « Cabilia i les eleccions algerianes », Europa de les Nacions, $\mathrm{n}^{\mathrm{0}}$ 71, 2009, p. 36-40.

Tilmatine M., « Berbère/Amazigh ou Kabyle ? Évolution et fluctuation d'une dénomination en contexte d'idéologies dominantes ", Quaderni di Studi Berberi e Libico-berberi (Studi Africanistici), $\mathrm{n}^{\mathrm{O}}$ 4, 2015, p. 387-414.

Tilmatine M., " Identidades y lenguas emergentes del Mediterráneo en el contexto de la crisis en Europa: el caso del amazige (bereber) en Cataluña », dans Nación $y$ Migración. España y Portugal frente a las migraciones contemporáneas, sous la dir. de Cornelia Siebert et al., Madrid, Biblioteca Nueva, 2015a, p. 261- 275.

Tilmatine M., «Arabization and Linguistic Domination: 
Berber and Arabic en the North of Africa ", dans Language Empires in Comparative Perspective : colonial and Postcolonial Linguistics, vol. 6, 2015, 1-17

ZouAïmia R., « L’introuvable pouvoir local », Insaniyat / [En ligne], no 16, 2002, URL : http://insaniyat.revues.org/7698 [consulté le 14/o6/2016].

\section{Notas}

1. Cet article s'inscrit dans le cadre des résultats de deux projets de recherche intitulés "Jeunesses, changement social, politique et sociétés en réseaux en Méditerranée : le cas des pays maghrébins » (CSO2011-29438-Co5-04) (2012-2014) et " Problèmes publics et militantisme au Maghreb : la participation sociale et politique des jeunes dans leur dimension locale et transnationale » (CSO2014-52998-C3-2-P) (2015-2017), financés par le ministère espagnol de l'Économie et de la Compétitivité.

2. J.G. Contamin, "Cadrages et luttes de sens », dans Penser les mouvements sociaux : conflits sociaux et contestations dans les sociétés contemporaines, sous la dir. de Olivier Fillieule et al., Paris, La Découverte, 2010, p. 57.

3. D. Snow, "Analyse des cadres et mouvements sociaux ", dans Les Formes de l'action collective, sous la dir. de Daniel Cefaï et Danny Trom, Paris, Éditions de l'EHESS, 2001, p. 8.

4. R. Benford, D.A. Snow, « Framing processes and social movements: an overview and assessment ", Annual review of Sociology, 26, 2000, p. 611-639.

5. B. Klandermans, " Mobilization and Participation : Social Psychological Expansions of Resource Mobilization Theory ", American Sociological Review, 49, 1984, p. 583-60o.

6. B. Klandermans, D. Oegema, "Potentials, Networks, Motivations, and Barriers: Steps Towards Participation in Social Movements ", American Sociological Review, 52, 1987, p. 519-531.

7. R. Benford et al., "Processus de cadrage et mouvements sociaux: présentation et bilan », Politix, 3/99, 2012, p. 217-255.

8. S. Chaker, "L'affirmation identitaire berbère à partir de 1900 : constantes et mutations (Kabylie) », Revue de l'Occident musulman et de la Méditerranée, 44/1, 1987, p. 13-34.

9. Cf. A. Guennoun, Chronologie du mouvement berbère 1945-1990, 
Alger, Casbah Editions, 1999, p. 21-26 ; A. Ouerdane, "Un conflit à plusieurs faces: "la crise berbériste" de 1949 " dans La Question berbère dans le mouvement national algérien, Québec, Sillery ; Paris, Septentrion, 1993.

10. M. Tilmatine, "Arabization and Linguistic Domination: Berber and Arabic en the North of Africa ", dans Language Empires in Comparative Perspective : Colonial and Postcolonial Linguistics, vol. 6, 2015, 1-17.

11. R.D. Benford et al., « Processus de cadrage et mouvements sociaux : présentation et bilan », p. 217-255.

12. R. Chaker, "Journal des événements de Kabylie (mars-mai 1980) », Les Temps modernes, $\mathrm{n}^{0}$ 432-433, juillet-août 1982, p. 383-438.

13. Ce discours se poursuivra, en revanche, sans discontinuer au Maroc. Voir l'interview de R. Raha par W. El Bouzdaini à dans l'hebdomadaire Maroc Hebdo du 23 au 29 octobre 2015, page 8, dans lequel Raha affirme que "l'amazigh est un patrimoine de tous les Marocains ». http://www.marochebdo.press.ma/rachid-rahalamazigh-est-un-patrimoine-de-tous-les-marocains/ [consulté le 24/10/2015].

14. Les deux documents sont disponibles sur différents sites kabyles. Voir par exemple http://soummam.o.s.f.unblog.fr/files/2009/04 /sminairedeyakourendossierculturelaout1980.pdf [consulté le 14/10/2015].

15. R. Bellil, S. Hachi, "Réflexions sur le mouvement culturel populaire en Algérie : la culture et le centralisme », 1981, paru dans la revue clandestine du Mouvement berbère Tafsut, série "normale" liée à l'actualité du terrain de lutte et lieu de réflexion sur le mouvement. Elle sera suivie, à partir de 1983, de la série Études et débats.

16. Ce concept est composé des termes global et local pour en faire un mélange (blend). " Nous savons également que le concept nous vient du Japon où l'agriculture a dû adapter des techniques globales aux conditions locales. Plus tard, le terme est passé aux affaires économiques. On parlera alors de "localisation globale", global localization, une perspective globale adaptée, là également, aux conditions locales. Cf. R. Robertson, «Glocalization : time and space and homogeneity-heterogeneity ", dans Global Modernities, sous la dir. de M. Featherstone et al., London, Thousand Oaks, New Delhi, Sage Publications, 1995, p. 28.

17. Le MCB se composait entretemps de deux branches principales : les Commissions nationales, proches du FFS et la Coordination nationale, proche du RCD auxquelles il fallait ajouter le Rassemblement national 
créé par Ferhat Mehenni, depuis son abandon du RCD. Cf. D. Abrous, "Le Haut Commissariat à l'Amazighité... », 1995, p. 584. Sur les enjeux des conversations et le retrait de la délégation des Commissions nationales des négociations, voir M. Tilmatine, « Berbère/Amazigh ou Kabyle ? Évolution et fluctuation d'une dénomination en contexte d’idéologies dominantes », Quaderni di Studi Berberi e Libico-berberi (Studi Africanistici), no 4, 2015, p. 387-414 2015.

18. D. Abrous, « Le Haut Commissariat à l'Amazighité ou les méandres d'une phagocytose », Annuaire de l'Afrique du Nord, 34, Paris, CNRS éditions, 1995, p. 583-590. 1995, p. 583-590.

19. A. Bouteflika, Discours à la Nation, Alger, 12 mars 2002, http://www.el-mouradia.dz/francais/president/recherche

/presidentrech.htm [consulté le 12/11/ 2015].

20. Ibid.

21. Ibid.

22. H. de Schutter, "The Linguistic Territoriality Principle - A critique », Journal of Applied Philosophy, 25/2, 2008, p. 105-120.

23. M. Issad, Rapport préliminaire de la Commission nationale d'enquête sur les événements de Kabylie (juillet 2001), 2001, p. 8, http://www.algeria-watch.de/farticle/revolte/issad_rapport.htm.

[consulté le 16/10/2015].

24. Ibid., p. 11.

25. http://www.maghress.com/fr/lobservateur/2300 : « Algérie : la Kabylie demande le divorce » ; http://www.lematindz.net /news/17254-kabylie-exister-en-dehors-de-la-dictature-

algerienne.html: « Kabylie : exister en dehors de la dictature algérienne » ; http://www.lefigaro.fr/international/2011/o7 /20/01003-20110720ARTFIG00524-en-algerie-la-kabylie-est-une-

poudriere.php : «Si le "divorce à l'amiable avec l'Algérie” séduit une frange importante de la jeunesse élevée dans le culte de la "résistance au pouvoir central”, l’idée est déjà plombée par le discours approximatif des dirigeants de ce mouvement qui tombent parfois dans la surenchère indépendantiste. Avec la proclamation, en juin 2010 à Paris, d'un "Gouvernement provisoire kabyle" en exil qui navigue à vue, sans ancrage dans le terroir, le MAK suscite la méfiance des militants les plus actifs. »

26. Voir, concernant le concept de hogra, A. Abderrahmane, La Hogra ou l'humiliation du peuple algérien, Montreuil, Babylone, 1992. Pour son usage en Europe on peut consulter V. Geisser, "Trop diplômés pour être honnêtes : la hogra des immigrés en "col blanc" », Migrations et société, vol. 138, $\mathrm{n}^{\circ}$ 6, 2011, p. 3-12. Ce concept, qui a été vulgarisé en Algérie dès l'indépendance du pays avec la célèbre formule 
de Ben Bella « Hagrouna », en référence à la « Guerre des sables » avec le Maroc, prendra plus de force lors des différentes révoltes qui secoueront le pays, en particulier lors des événements dits du « Printemps noir de Kabylie ». Des photos de manifestantes et de manifestants portant des pancartes avec ce concept - en version arabe ou kabyle : tamehqranit - ont circulé sur la toile et l'ont largement divulgué. Ce terme, d'usage courant dans les parlers d'Afrique du Nord, s'est entretemps complètement établi dans les sciences sociales et dépasse même le cadre nord-africain pour s'appliquer dans d'autres pays africains comme le Burkina-Faso. Voir à cet effet L. Chouli, « Les mouvements sociaux de 1998 et 2011 au Burkina Faso comme indices et réponses à la crise de la représentation politique », Le Retour de la question politique : crise de la représentation et luttes démocratiques en Afrique, $4^{\mathrm{e}}$ Colloque international de Dakar, 22-24 mai 2013 (à consulter sur essai.gabrielperi.fr/IMG/pdf/7-lila_chouli.pdf).

Concernant le Maroc, cf. par exemple S. Bennis, "Société civile et nouveaux paradigmes conceptuels : le concept de hogra », à consulter sur le site du Centre d'études et de recherches en sciences sociales, http://www.cerss__ ma.org/new/index.php?option=com content\& view=article\&id=279: societe-civile-et-nouveaux-paradigmesconceptuels--le-concept-de-l-hogra-r\&catid=86:article-bennis\& Itemid=108, mercredi 9 janvier 2013.

27. Professeur agrégé de droit, spécialiste du droit international, Issad est décédé à Paris le 27 avril 2011. Il avait occupé, en 1999, le poste de président de la Commission nationale de réforme judiciaire (CNRF) avant d'être désigné par le président Bouteflika pour diriger la commission d'enquête sur les événements de Kabylie en 2001.

28. Le concept $d^{\prime}$ ' « autonomie personnelle » renvoie dans le texte du FFS aux droits individuels en général et au respect des droits de l'homme, du droit de sûreté, de mouvement, de l'intégrité corporelle, de ses opinions, de ses qualités imaginatives, de son travail artistique, de sa langue maternelle » (p. 16). Le concept d' « autonomie locale » tel que défendu par le document du FFS préconise de revenir aux djemâa qui ont permis « à notre Nation et à notre culture de survivre à travers les âges ». Les djemâa devraient jouir de pouvoirs étendus, hors des contraintes tutélaires de l'administration centrale (p. 17-18). Enfin, les institutions de l'autonomie locale et de " l'autonomie régionale » doivent selon le FFS « résulter d'élections libres [...]. C'est le peuple qui doit choisir et éventuellement congédier ses mandataires aussi bien au niveau local qu'au niveau régional », (p. 19). Cf. Front des forces socialistes, « L'alternative démocratique révolutionnaire à la catastrophe nationale », 1979.

29. M. Moffok, « Le RCD relance son projet de régionalisation positive », Impact24.Info du 3/07/2015, [en ligne] URL : 
http://www.impact24.info/le-rcd-relance-son-projet-deregionalisation-positive/ [consulté le 12/06/2016].

30. Voir par exemple les positions publiques de certains intellectuels kabyles, notamment celles de S. Chaker, figure prééminente du Mouvement berbère et professeur de linguistique berbère à l'INALCO (Paris). Certaines prises de position à cet égard sont publiées par exemple dans La Question amazighe : interrogations actuelles..., Actes de la table ronde organisée par le MCB-France, Paris 21 avril 1996, ou dans ce qui pourrait être considéré comme le premier résultat d'une réflexion commune sur l'autonomie de la Kabylie, Kabylie : l'autonomie en débat, Actes du séminaire d'Ecancourt, France, 1-3 mars 2002, Paris.

31. Une première rencontre Kabylie-Catalogne a eu lieu à Barcelone au mois de septembre 2002 et a rassemblé des militants et des hommes politiques kabyles et catalans. Les travaux de cette rencontre ont été édités par C. Castellanos, S. Chaker, M. Tilmatine, Actes de la rencontre Kabylie-Catalogne : identités nationales et structures étatiques dans le contexte méditerranéen, Barcelone, 13 et 14 septembre 2002, Paris, Editions berbères, 2008.

32. C. Canut, "À la frontière des langues : figures de la démarcation », Cahiers d'études africaines, $\mathrm{n}^{0}$ 163-164, XLI-3-4, 2001, p. 443-463.

33. M. Tilmatine, "L'Etat-Nation face à la revendication berbère : quel(s) modèle(s) pour l'Algérie ? », Actes de la table ronde organisée par le MCB-France, 21 avril 1996, Paris, MCB-France, 1996a, p. 33-44, ou C. Castellanos et al., Actes de la rencontre Kabylie-Catalogne, Barcelone, 13 et 14 septembre 2002, Paris, Editions berbères, 2008.

34. S. Chaker, "Pour l'autonomie linguistique de la Kabylie », Le Monde, 11 juillet 1998.

35. M. Tilmatine, « Identidades y lenguas emergentes del Mediterráneo en el contexto de la crisis en Europa: el caso del amazige (bereber) en Cataluña », dans Nación y Migración. España y Portugal frente a las migraciones contemporáneas, sous la dir. de Cornelia Siebert et al., Madrid, Biblioteca Nueva, 2015a, p. 261- 275.

36. Créé en 2004 par un certain nombre d'intellectuels kabyles à Paris qui avaient déjà organisé des rencontres sur l'autonomie de la Kabylie et qui ont participé à la publication de deux ouvrages sur le sujet ( $L a$ Question amazighe : interrogations actuelles... ainsi que Kabylie : l'autonomie en débat). Un forum avait été créé à cet effet sur une page kabyle : https://www.kabyle.com/archives/trier-l-info-kabyle/breve /le-cercle-d-etude-et-de-reflexion.

37. O. Ilikoud, «FFS et RCD : partis nationaux ou partis kabyles ? ", Revue du monde musulman et de la Méditerranée, 111-112, mars 
2006, mis en ligne le 8/12/ 2011, URL : http://remmm.revues.org/2870 [consulté le 14/o7/2015].

38. Cf. http://www.makabylie.org/index.php/le-mak-en-quelquesquestions/.

39. F. Mehenni, Algérie : la question kabyle. Essai, Paris, Editions Michalon, 2004, p. 141.

40. R. Zouaïmia, « L’introuvable pouvoir local », Insaniyat, $\mathrm{n}^{\circ} 16$, 2002, p. 61.

41. Cf. Contamin, « Cadrages et luttes de sens », p. 58.

42. Voir notamment les grandes manifestations qui ont eu lieu dans plusieurs villes européennes et d'Amérique du Nord. Voir les informations rapportées à cet effet dans différents médias, par exemple: http://algeriefranceinfos.com/la-marche-du-mak-a-parisen-images/ ou bien le texte de Maglor.fr (Médias des Maghrébins du monde), http://www.maglor.fr/maglor/index.php?option=com_k2\& view=item\&id=8713:algerie-le-mak-mobilise-avec-succes-memea-montreal\&Itemid $=132$.

43. Projet pour l'autonomie de la Kabylie (PAK) du mardi 27 janvier 2009. Amendé et adopté au $2^{\mathrm{e}}$ congrès du MAK, à Sahel, At Wizgan, les 9 et 10 décembre 2011. http://www.makabylie.info /spip.php?article62. Le projet n'a pas été publié par les journaux algériens, mais il est disponible sur divers sites Internet kabyles comme par exemple www.kabyle.com ou sur la page du MAK : www.makabylie.info/ahric7.

44. Source : http://www.makabylie.info/?article1341.

45. A. Hammouche, « La Kabylie, la démocratie et l'autonomie », quotidien Le Matin, 22 avril 2010, [en ligne] URL: http://www.lematindz.net/news/3067-la-kabylie-la-democratie-etlautonomie-par-mustapha-hammouche.html [Consulté le 16/10/2015].http://www.lematindz.net/news/3067-la-kabylie-lademocratie-et-lautonomie-par-mustapha-hammouche.html; ou bien dans El Watan, comme dans le numéro du 20 avril 2015, [en ligne] URL : $\quad$ http://www.elwatan.com/dyn/imprimer.php?link=http \%3A\%2F\%2Fwww.elwatan.com\%2Factualite\%2Fprintemps-berberea-tizi-ouzou-des-milliers-de-manifestants-dans-les-

rues-20-04-2015-292859_109.php ou du 20 avril 2014, http://elwatan2014.com/ar/item/1777-Le-MAK-et-les-\%C3 \%Agtudiants-ont-march\%C3\%A9-ensemble-\%C3\%Ao-Bouira ou de manière générale dans le journal Algérie Focus du 28 avril 2014 : http://www.algerie-focus.com/blog/2014/o4/revue-de-presse-succesde-la-marche-du-mak-a-tizi-ouzou-du-tout-repressif-a-la-totaletolerance/. 
46. Cf. le quotidien El-Khabar du 14 avril 2016, qui titre: " Le MAK fait-il peur au gouvernement? ", [en ligne] URL: http://www.elkhabar.com/press/article/104138 /\#sthash.gh6T2Fvg.cmWRytzx.dpbs.

47. Plusieurs documents justifient et reprennent ce discours au sein du MAK. Cf. par exemple le document intitulé : « Demande officielle d'un statut d'autonomie pour la Kabylie » adressé à la Présidence de la République algérienne, au Gouvernement algérien, à l'Assemblée populaire algérienne, au Sénat algérien et au Conseil constitutionnel algérien, avec copie à différentes organisations internationales. Document du 5 juin 2008. Voir également le texte du Projet pour un État kabyle (PEK) adopté à la conférence nationale des cadres du MAK le 24 janvier 2014 à Smaoun (Bejaïa), point $n^{0} 8$.

48. Cf. http://www.siwel.info/communique-du-mouvement-pourl-autodetermination-de-la kabylie a $5585 . h \mathrm{html}$.

49. http://www.elwatan.com/actualite/progression-du-mak-le-ffsaccuse-le-pouvoir-23-04-2016-319400_109.php.

50. http://www.la-clau.net/noticia/el-partido-popular-fabrica-desindependentistes-catalans-10786

51. http://www.maglor.fr/maglor/index.php?option=com_k2\& view=item\&id=8713:algerie-le-mak-mobilise-avec-succes-memea-montreal\&Itemid $=132$

http://algeriefranceinfos.com/la-marche-du-mak-a-paris-en-images/

https://www.youtube.com/watch?v=swLtkulBFro

52. http://www.libe.ma/Pourquoi-ne-pas-appliquerl-autodetermination-a-la-Kabylie-et-aux-Touaregs_a37667.html

53. http://www.siwel.info/Declaration-de-l-Anavad-le-royaume-dumaroc-reconnait-le-droit-du-peuple-kabyle-a-sonautodetermination_a7968.html

54. http://archives.siwel.info/Discours-historique-du-president-del-Anavad-devant-l-Assemblee-generale-du-MAK-Anavad-a-Montreuille-25-09-2016_a9783.html [consulté le 01/03/2017].

55. http://www.lematindz.net/news/22329-bouaziz-ait-chebibdemissionne-de-la-presidence-du-mak.html

56. https://www.parlementkabyle.com/index.php/parlement-kabyle /projet-parlement [consulté le 01/03/2017].

57. http://reseau-anavad.com/nominations-abrogationsdecisions-465 [consulté le 01/03/2017].

58. http://www.elwatan.com/actualite/le-rpk-nouveau-mouvementautonomiste-pour-la-kabylie-26-02-2017-340040_109.php [consulté 
le $01 / 03 / 2017]$.

59. https://www.kabyle.com/breves/manifeste-reconnaissanceconstitutionnelle-dun-statut-politique-particulier-kabylie-23828

[consulté le 01/03/2017].

60. http://www.elwatan.com/actualite/pour-nous-la-kabylie-faitpartie-de-l-algerie-et-doit-y-rester-27-02-2017-340126_109.php [consulté le 01/o3/2017].

61. http://www.lexpress.fr/actualites/1/monde/l-algerie-consacre-lalangue-berbere-apres-une-longue-lutte_1761276.html

62. Voir le texte du quotidien Le Soir d'Algérie sur Ferhat: http://www.lesoirdalgerie.com/articles/2015/07/01/print-

16-180714.php ou sur Bouaziz Chebib du quotidien algérien Tout sur l'Algérie (TSA): http://www.tsa-algerie.com/20150420/entretienavec-bouaziz-ait-chebib-president-du-mak/ ou enfin un entretien avec Ferhat sur ses projets politiques dans le journal Focus Algérie du 19 juillet $2015:$ : http://www.algerie-focus.com/blog/2015 /07/independance-de-la-kabylie-sa-nationalite-algerienne-etevenement-de-ghardaiaferhat-mehenni-nous-dit-tout/

63. http://www.mondeberbere.com/rebonds/algeriefed.htm. L'auteur de cette proposition de création d'un parti politique, le Rassemblement pour l'Algérie algérienne fédérale, est également un vieux militant kabyle, R. Ali-Yahia : http://site.raaf.free.fr/.

64.

http://www.lesoirdalgerie.com/articles/2015/07 /28/article.php?sid=181992\&cid=2

65. http://www.elwatan.com/actualite/il-faut-reconnaitre-lemouvement-pour-l-autonomie-de-la-kabylie18-11-2014-278167_109.php

66. http://www.elwatan.com/actualite/idir-defend-le-droit-de-ferhata-s-exprimer-01-10-2013-229881_109.php

67. S. Haidar, "Culture : AD Gladium Ferhat. Parlons-en! ", Le Soir d'Algérie, $1^{\text {er }}$ juillet 2015. http://www.lesoirdalgerie.com/articles /2015/07/01/print-16-180714.php;___ http://www.lesoirdalgerie.com /articles/2015/07/01/article.php?sid=180714\&cid=16 [consulté le 7/06/2015].

68. N. Boukrouh, « Une fausse nation », Le Soir d'Algérie, 9 juin 2016, [en ligne] URL: http://www.lesoirdalgerie.com/articles/2016/o6 /09/article.php?sid=197577\&cid=41 [consulté le 12/05/2016].

69. https://www.youtube.com/watch?v=zmua7oBSJuE

70. S. Haidar, op. cit.

71. http://www.elwatan.com/actualite/le-probleme-fondamental-del-algerie-c-est-de-refonder-la-nation-27-02-2017-340115_109.php 
[consulté le 2/03/2017].

72. http://lexalgeria.free.fr/politiq.htm

73. http://www.msnfcf.gov.dz/fr/public_file

/document_1398811133.pdf

74. http://fr.africatime.com/maurice/articles/libye-les-autonomistesde-lest-ne-reconnaissent-pas-le-nouveau-premier-ministre; voir également https://www.kabyle.com/articles/imazighen-libye-voielautodetermination-22047-14082013 ou bien http://www.tabrat.info $/ ? \mathrm{p}=4865$.

75. Cf. M.R. De Madariaga, España y el Rif. Crónica de una historia casi olvidada, Melilla, La biblioteca de Melilla, tercera edición, 2008.

76. http://www.alhoceimaonline.com/online/index.php/video /videosport/299-le-mouvement-de-lautonomie-du-rif-se-reunita-alhoceima. Le mouvement autonomiste du RIF revendique le Rifain contre l'amazigh standard: http://www.siwel.info/Le-Mouvementpour-l-autonomie-du-RIF-appelle-a-la-sauvegarde-de-la-languerifaine-et-a-ne-pas-succomber-au-slogan-de_a7321.html.

77. http://telquel.ma/2017/05/29/nasser-zefzafi-arrete-pour-atteintea-la-securite-interieure-de-letat_1548409

Autor

Mohand Tilmatine

\section{Professeur à l'université de Cadix} (Espagne) dans la spécialité « Études berbères ». Il dirige également depuis 2000 le groupe de recherche HUM 685 Langues et sociétés arabes et berbères (http://www.uca.es/gruposinv/HUM683/). Ses axes de recherche en sciences humaines et sociales couvrent la question 


\section{des minorités et des revendications identitaires berbères en Afrique du Nord et dans la diaspora en Europe.}

(C) Centre Jacques-Berque, 2017

Condiciones de uso: http://www.openedition.org/6540

Referencia electrónica del capítulo

TILMATINE, Mohand. Des revendications linguistiques aux projets d'autodétermination : le cas de la Kabylie (Algérie) In: Les revendications amazighes dans la tourmente des « printemps arabes »: Trajectoires historiques et évolutions récentes des mouvements identitaires en Afrique du Nord [en línea]. Rabat: Centre Jacques-Berque, 2017 (generado el 21 febrero 2018). Disponible en Internet: <http://books.openedition.org/cjb/1359>. ISBN: 9791092046335. DOI: 10.400o/books.cjb.1359.

Referencia electrónica del libro

TILMATINE, Mohand (dir.) ; DESRUES, Thierry (dir.). Les revendications amazighes dans la tourmente des " printemps arabes »: Trajectoires historiques et évolutions récentes des mouvements identitaires en Afrique du Nord. Nueva edición [en línea]. Rabat: Centre Jacques-Berque, 2017 (generado el 21 febrero 2018). Disponible en Internet: <http://books.openedition.org /cjb/1299>. ISBN: 9791092046335. DOI: 10.400o/books.cjb.1299.

Compatible con Zotero 